\title{
Notarzt und Sozialnot
}

$D_{i \text { ie }}$ Notfallmedizin befindet sich im Umbruch. Fraglos wird ihr Repertoire auch künftig die Basis des notärztlichen Handelns bleiben und zu den unverzichtbaren Kernkompetenzen des Notarztes zählen. Die derzeitige tägliche Praxis zeigt jedoch deutlich, dass sich Notfallmedizin nicht ausschließlich auf die Behandlung kompromittierter Vitalfunktionen reduzieren lässt. Besonders in Großstädten und Ballungsräumen weist sie - von den Leitstellen vermittelt dem Notarzt neue, von der ursprünglichen Aufgabenstellung abweichende Funktionen zu, die ursprünglich den KV-Vertragsärzten zugeordnet waren. Der Notarzt wird als überall verfügbare und niederschwellig erreichbare ärztliche Institution zunehmend auch mit primär nicht lebensbedrohlichen Krisensituationen unterschiedlichster Art konfrontiert. Vielerorts prägen bereits heute Notfälle, die durch das Versagen sozialer Ressourcen in Familie, Umfeld oder Gesellschaft entstanden sind, sowie psychiatrische Erkrankungen die Einsatzrealität. Es ist unübersehbar, dass der gesellschaftliche Auftrag weit über ihre ursprüngliche Konzeption - nämlich die Sicherung und Wiederherstellung von Vitalfunktionen in lebensbedrohlichen Situatio- nen - hinausgeht. Der Widerspruch von tradiertem Selbstverständnis und erlebter Wirklichkeit lässt ein für jeden Rettungsdienstmitarbeiter wahrnehmbares Spannungsfeld entstehen.

Zwar lässt sich diskutieren, ob diese Aufgaben nicht eigentlich in der Verpflichtung der Vertragsärzte der KV liegen und daher dorthin zurückzuweisen sind. Um jedoch der Notfallmedizin ihre Schlüsselfunktion im Gesamtkonzept der Akutversorgung zu erhalten, erscheint es unumgänglich, bisherige Konzepte und Strukturen zu überdenken und gegebenenfalls zu ergänzen. Zur Weiterbildung in der Notfallmedizin ist dazu die Aufnahme eines Dialogs mit Epidemiologen, Medizinsoziologen und Public-Health-Forschern unerlässlich. Der einzelne Notarzt muss über ausreichendes sozialmedizinisches Basiswissen verfügen, um seinen individualmedizinischen Auftrag adäquat erfüllen zu können.

"Der Notarzt wird zunehmend mit Krisensituationen unterschiedlichster Art konfrontiert."

Im Gegenzug könnte der zeitnah verfügbare Datenpool des Systems Rettungsdienst für Zwecke der PublicHealth-Forschung nutzbar gemacht werden: die Analyse von Einsatzmustern als sensitiver Indikator für soziale Veränderungen oder zur Überprüfung der Effektivität regionaler medizinischer und sozialer Versorgungssysteme.
Auf einem interdisziplinären Workshop, der unter dem Motto „Notarzt und Sozialnot - der gesellschaftliche Auftrag der Notfallmedizin" stand und im Dezember 2001 in München stattfand, wurde die soziale Dimension der Notfallmedizin unter den verschiedensten Gesichtspunkten erörtert. Auf der Tagesordnung stand neben notfallmedizinisch relevantem epidemiologischem Basiswissen auch der Notarzteinsatz mit primär psychosozialen Problemstellungen. Alte und Alleinstehende, Suchtkranke, Menschen ohne festen Wohnsitz, Migranten, Asylsuchende und ethnische Minderheiten, Arbeitslose und in Armut lebende Menschen stellen wesentliche Risikopopulationen für die Inanspruchnahme akutmedizinischer Leistungen dar.

Einige auf diesem Workshop diskutierte Themen sind in dem vorliegenden Heft von Notfall- und Rettungsmedizin ausführlich dargestellt.

\section{Madler}

\footnotetext{
(C) Springer-Verlag 2002

Prof.Dr.Christian Madler Institut für Anaesthesiologie und Notfallmedizin I,Westpfalz-Klinikum GmbH, Hellmut-Hartert-Str. 1, 67655 Kaiserslautern
} 